\title{
Inmunoexpresión de p53 y su relación con el grado de severidad en displasia epitelial oral.
}

\author{
Immunoexpression of p53 and its relationship with the \\ degree of severity in oral epithelial dysplasia.
}

\author{
Elizabeth Barbarita Narváez García,* Elba Rosa Leyva-Huerta, ${ }^{\ddagger}$ Javier Portilla-Robertson, $§$ \\ Blanca Itzel Mendoza-Espinosa, ${ }^{\rrbracket}$ Adriana Molotla-Fragoso,, Luis Fernando Jacinto-Alemán ${ }^{\ddagger}$
}

\section{RESUMEN}

Introducción: La displasia epitelial oral (DEO) es la presencia de alteraciones celulares y tisulares, lo que puede significar una etapa anterior al desarrollo del cáncer. Múltiples marcadores han sido considerados para estimar su potencial neoplásico y evolución a carcinoma, incluyendo a la molécula p53, se considera como participe de diversos fenómenos de la homeostasis celular. Objetivo: Determinar la relación entre la inmunoexpresión de p53 DO-7 y PAb 240 con el grado de severidad de la displasia epitelial oral. Material y métodos: Se analizaron nueve muestras de DEO (tres para cada grado de severidad). La inmunoexpresión de p53 tipo silvestre (DO-7) y forma mutada (PAb 240), fue determinada a través de ensayo de inmunohistoquímica por peroxidasa. Se obtuvieron la media y desviación estándar y se realizó la prueba $\chi^{2}(\mathrm{p}<0.05)$. Resultados: La edad media fue de $65.7 \pm 11.4$ años, la zona anatómica con mayor presencia de DEO es el borde lateral de la lengua. Ocho de nueve muestras fueron positivas para DO-7 y solo dos para PAb 240. Conclusiones: Nuestros resultados indican que, aunque la expresión de p53 DO-7 podría estar relacionada parcialmente con la patogénesis de la displasia epitelial, no todas las displasias presentaron la forma mutada de p53 (PAb 240). Lo cual coincide con el comportamiento biológico incierto de las displasias al poder permanecer sin cambios, involucionar o transformarse.

Palabras clave: Displasia epitelial, p53, inmunoexpresión, transformación.

\section{ABSTRACT}

Introduction: Oral epithelial dysplasia (OED) is the presence of cellular and tissue alterations, which may mean a stage prior to the development of cancer. Multiple markers have been considered to estimate its pathogenic potential and evolution to neoplasms, including the p53 molecule, considered as participating in various phenomena of cellular homeostasis. Objective: To determine the relationship between the immunoexpression of p53 DO-7 and PAb 240 with the degree of severity of oral epithelial dysplasia. Material and methods: Nine OED samples were analyzed (three for each degree of severity). The immunoexpression of wild-type p53 (DO-7) and mutated form (PAb 240) was determined through a peroxidase immunohistochemical assay. The mean and standard deviation were obtained, and $\chi^{2}$ test ( $p$ $<0.05)$ were performed. Results: The mean age was $65.7 \pm 11.4$ years, with a greater presence of $O E D$ in the anatomical area of the lateral side of the tongue. Eight out of nine samples were positive for DO-7 and only two for PAb 240. Conclusions: Our results indicate that, although the expression of $553 \mathrm{DO}-7$ could be partially related to the pathogenesis of epithelial dysplasia, not all dysplasias presented the mutated form of $p 53$ (PAb 240), which coincides that not all dysplasias have a potential for malignant transformation and that could be related to other oncogenic mechanisms.

Keywords: Epithelial dysplasia, p53, immunoexpression, transformation.

\footnotetext{
* Cirujana Dentista. Estudiante de la Especialidad de Odontopediatría, Universidad Latinoamericana, campus Cuernavaca. México.

‡ Doctor en Ciencias. Profesor del Departamento de Medicina Oral y Patología, División de Postgrado e Investigación, Facultad de Odontología, Universidad Nacional Autónoma de México. México.

$\S$ Doctor en Ciencias. Coordinador del Departamento de Medicina Oral y Patología, División de Postgrado e Investigación, Facultad de Odontología, Universidad Nacional Autónoma de México. México.

" Especialista en Periodontología. Profesora de Periodontología, Facultad de Odontología, Universidad Nacional Autónoma de México. México. \| Maestra en Ciencias. Profesora del Departamento de Medicina Oral y Patología, División de Postgrado e Investigación, Facultad de Odontología, Universidad Nacional Autónoma de México. México.
}

Recibido: 09 de agosto de 2021. Aceptado: 20 de septiembre de 2021.

Citar como: Narváez GEB, Leyva-Huerta ER, Portilla-Robertson J, Mendoza-Espinosa BI, Molotla-Fragoso A, Jacinto-Alemán LF. Inmunoexpresión de p53 y su relación con el grado de severidad en displasia epitelial oral. Rev ADM. 2021; 78 (5): 258-263. https://dx.doi.org/10.35366/102032

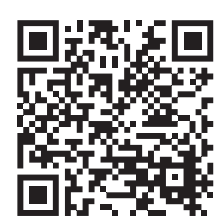




\section{INTRODUCCIÓN}

L os trastornos orales potencialmente malignos (TOPM) incluyen una variedad de lesiones y afecciones del epitelio oral que tienen un mayor riesgo de transformación maligna; éstos incluyen a la leucoplasia, eritroplasia, fibrosis submucosa y liquen plano. Cada TOPM tiene diferentes características clínicas; sin embargo, en la actualidad la detección histológica de displasia epitelial oral (DEO) es el indicador más útil asociado a la posible transformación maligna, lo cual obliga el realizar un seguimiento prolongado del paciente. ${ }^{1}$ La DEO puede clasificarse como leve, moderada y severa utilizando criterios diagnósticos para cuantificar cambios arquitectónicos y citológicos. ${ }^{2,3}$ Uno de los hallazgos más relevantes respecto a su asociación con la patogénesis del carcinoma oral de células escamosas (COCE) es su no linealidad, es decir, no siempre toda displasia severa fue precedida por una moderada y no es necesario que un COCE sea precedido por una displasia severa, aunque aproximadamente $80 \%$ de los carcinomas orales se encuentran relacionados con algún TOPM. ${ }^{4}$

La evidencia indica que la transición de un epitelio normal hacia un TOPM y/o COCE es el resultado de la acumulación de alteraciones genéticas, epigenéticas e inestabilidad genómica. La proliferación celular sostenida, evasión de la supresión del crecimiento, resistencia a la apoptosis, inmortalidad replicativa, angiogénesis, invasión y metástasis, así como la reprogramación del metabolismo energético, evasión de la vigilancia inmunitaria, inestabilidad genómica e inflamación son aspectos fundamentales de la biología neoplásica y de los TOPM. ${ }^{5}$ Dentro de las moléculas más importantes reportadas en el proceso de la carcinogénesis se encuentra p53, esta molécula participa en mecanismos de respuesta al estrés celular, inducción de la detención del ciclo celular, apoptosis, senescencia, reparación del ADN o cambios en el metabolismo. ${ }^{6}$ Se ha reportado que la proteína p53 puede localizarse en diferentes TOPM e incluso relacionarse con el grado de displasia. ${ }^{7,8} \mathrm{No}$ obstante, es importante señalar que esta molécula tiene un tiempo de vida corto y puede presentar diferentes cambios conformacionales e incluso mutaciones, los cuales pueden ser detectados por distintos métodos como la inmunohistoquímica. ${ }^{9}$ Nuestro objetivo fue determinar la relación de la inmunoexpresión p53 (DO-7 convencional o wild-type) y p53 mutado (PAb 240) con el grado de severidad de la DEO.

\section{MATERIAL Y MÉTODOS}

Población y tipo de estudio: se realizó un estudio piloto observacional analítico utilizando nueve muestras de displasia oral. Los especímenes fueron colectados aleatoriamente del archivo del Servicio de Diagnóstico Histopatológico del Departamento de Patología Oral y Medicina (ISO-9001:2015 certificado CMX C SGC 157 2017). Acorde con el aviso de privacidad de la Facultad de Odontología, UNAM, los datos obtenidos fueron empleados con la finalidad de difundir el conocimiento científico, respetando el anonimato del paciente y expediente clínico. ${ }^{10}$ Sólo se registró la edad, sexo, zona anatómica y grado de displasia de cada uno de los casos. El grado de displasia fue reevaluado por dos patólogos bucales.

Ensayo e interpretación de inmunohistoquímica: se realizó en ensayo de inmunohistoquímica por peroxidasa de acuerdo con lo reportado. ${ }^{11}$ De cada muestra, se obtuvieron cuatro laminillas tratadas con poli-L-lisina con secciones de $4 \mu \mathrm{m}$. Las muestras fueron desparafinadas y rehidratadas convencionalmente en lavados de xilol y alcohol. La recuperación antigénica se realizó con un buffer de citrato de $10 \mathrm{mM}$ en ebullición mediante microondas (600 W durante 3 minutos y 30 segundos). Se inhibió peroxidasa endógena con peróxido de hidrógeno a 3\% durante 20 minutos (Sigma-Aldrich, St. Louis, MO, USA). El fondo inespecífico fue bloqueado con $100 \mu \mathrm{L}$ de albúmina a $2 \%$ durante 20 minutos (Sigma-Aldrich, St. Louis, MO, USA), y después con solución de Triton X-100 a 0.2\% durante 20 minutos (Sigma-Aldrich, St. Louis, MO, USA). Se incubó con el anticuerpo primario para la forma convencional (monoclonal de ratón, DO-1, sc-126) y mutada de p53 (monoclonal de ratón, PAb 240, sc-99); ambas diluciones fueron ajustadas a 1:200 e incubando durante toda la noche a $4{ }^{\circ} \mathrm{C}$. Los controles negativos se obtuvieron mediante la omisión del anticuerpo primario, sustituyéndolo por tampón de fosfato salino (phosphate-buffered saline, PBS).

Terminada la incubación, se lavó con TBS (solución Tris buffer) por tres minutos en tres ocasiones. Después se incubó con Super-Enhancer (BioGenex, QD430-XAKE, Fremont, CA) durante 20 minutos, para después lavar con TBS. Se incubó con biotin-link HRP (BioGenex, QD430XAKE) por 30 minutos. Se lavó con TBS y enseguida se reveló con diaminobencidina (DAB, BioGenex, QD430XAKE) durante cinco minutos. Se lavó con agua bidestilada durante tres minutos, contratiñendo con hematoxilina de Harris durante dos minutos. Se lavó con agua corriente para eliminar el excedente, para después realizar la deshidratación y aclarado en inmersiones de alcohol y xilol, montando el cubreobjetos con resina hidrofóbica.

Para el análisis de cada marcador, se obtuvieron cinco fotomicrografías de campos a 400 aumentos utilizando el microscopio Leica DM750, la cámara ICC50 HD y el software LAS EZ 3.0 (Leica Microsystems, Suiza). 
La intensidad de inmunoexpresión fue expresada en unidades ópticas (uo) empleando el software Image J $(\mathrm{NIH}$, Bethesda MD, USA), calibrando la cuantificación para establecer la escala de: 0-0.9/negativo, 1-1.9/leve, 2-2.9/moderada y > 3/elevada. La proporción de células positivas fue establecida semicuantitativa considerando la proporción media de los cinco campos analizados para categorizar como: 0) ausencia; 1) 1 a 10\%; 2) 11 a 50\%; y 3 ) superior a $50 \%$.

Análisis estadístico: se realizó el análisis descriptivo de las variables mencionadas, obteniendo la media y la desviación estándar para la edad e intensidad de inmunoexpresión, además de la prueba de $\chi^{2}$ para la intensidad y proporción de células positivas considerando un valor $p<0.05$ como significativo, empleando el software SPSS (versión 22, IBM SPSS, IL, USA).

\section{RESULTADOS}

Características clínico-demográficas: la edad media fue de $65.7 \pm 11.4$ años con un intervalo de 48-86 años. Cinco especímenes eran de pacientes varones, y cuatro provenían de pacientes femeninos. La distribución de la zona anatómica fue la siguiente: cinco especímenes del borde lateral de la lengua; uno de la cara ventral de lengua; uno de paladar duro; y dos de encía insertada (Tabla 1). Respecto al grado de displasia y su relación con la zona anatómica y el género, se observó una distribución heterogénea.

Ensayo de inmunohistoquímica: el análisis inmunohistoquímico indicó que p53 DO-7 fue positivo en ocho de nueve muestras. De esas ocho muestras positivas, la intensidad de inmunoexpresión observada fue predominantemente leve en estrato basal $(n=5)$, una moderada y dos elevadas. Respecto a la proporción de células positivas, cinco muestras presentaron un rango de 1-10\% y tres muestras un rango superior a $50 \%$ de células positivas. Tanto la intensidad como la proporción de células positivas no presentaron relación estadísticamente significativa con el grado de displasia. La forma mutada PAb 240 fue positiva sólo en dos especímenes de los positivos a la forma convencional, ambos de severidad moderada (Figura 1). La intensidad de inmunoexpresión fue leve para ambas con una proporción dentro del rango de 1-10\%, de igual forma no se observó significancia entre estas variables con la severidad de displasia (Tabla 2).

\section{DISCUSIÓN}

Por lo general, la idea más aceptada es que la identificación microscópica de las DEO es el predictor más fuerte de la transformación maligna de algún TOPM. Sin embargo, se ha considerado que la combinación de características clínicas, histopatológicas y moleculares son necesarias para mejorar dicha predicción. ${ }^{12}$

Una variable considerable para el desarrollo de estos trastornos es la edad, existen reportes que mencionan en relación con el envejecimiento que la mucosa oral sufre cambios que la hacen más susceptible a factores irritativos, y que los daños adquiridos a lo largo de la vida pueden hacerse presentes. ${ }^{13}$ Esto hace considerar que el riesgo a desarrollar cáncer aumenta a partir de la quinta a sexta década de la vida, lo cual coincide con la edad media observada en este estudio.

Tabla 1: Distribución clínica demográfica.

\begin{tabular}{|c|c|c|c|c|}
\hline & \multicolumn{4}{|c|}{ Displasia epitelial oral, n (\%) } \\
\hline & Leve & Moderada & Severa & Total \\
\hline \multicolumn{5}{|l|}{ Género } \\
\hline Masculino & $2(22.2)$ & $1(11.1)$ & $2(22.2)$ & $5(55.5)$ \\
\hline Femenino & $1(11.1)$ & $2(22.2)$ & $1(11.1)$ & $4(44.5)$ \\
\hline Edad (años), media $\pm \mathrm{DE}$ & $57 \pm 9.5$ & $63.3 \pm 7.5$ & $54.6 \pm 8.08$ & $65.7 \pm 11.4$ \\
\hline \multicolumn{5}{|l|}{ Zonas anatómicas } \\
\hline Borde lateral de la lengua & $1(11.2)$ & $2(22.2)$ & $2(22.2)$ & $5(55.6)$ \\
\hline Cara ventral de la lengua & 0 & 0 & $1(11.1)$ & $1(11.1)$ \\
\hline Paladar & $1(11.1)$ & 0 & 0 & $1(11.1)$ \\
\hline Encía insertada & $1(11.1)$ & $1(11.1)$ & 0 & $2(22.2)$ \\
\hline
\end{tabular}



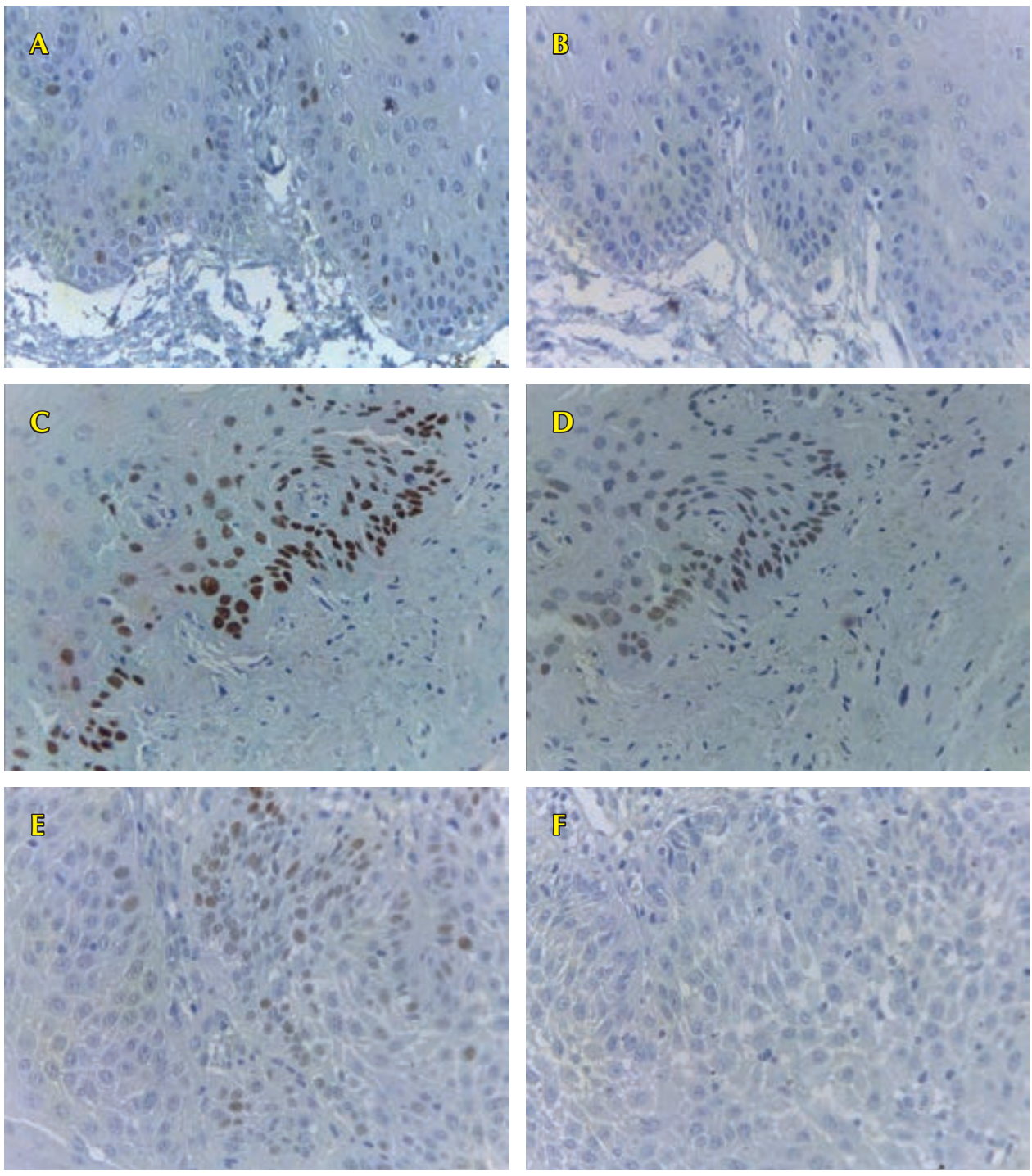

Figura 1:

Análisis de inmunoexpresión. A) Inmunoexpresión positiva de p53 DO-7 en displasia epitelial oral (DEO) leve. B) Inmunoexpresión negativa de p53 PAb-240 en DEO leve. C) Inmunoexpresión positiva de p53 DO-7 en DEO moderada. D) Inmunoexpresión positiva de p53 PAb-240 en DEO moderada. E) Inmunoexpresión positiva de p53 DO-7 en DEO severa. F) Inmunoexpresión negativa de p53 PAb-240 en DEO severa. Fotomicrografías a 400×.
De manera tradicional, se ha considerado que tanto la displasia oral como los carcinomas se presentan con mayor frecuencia en el género masculino. ${ }^{14}$ Sin embargo, se ha reportado que esta tendencia ha cambiado en tiempos recientes, lo cual puede atribuirse a cambios conductuales respecto a la exposición a los factores de riesgo tradicionales en el género femenino. ${ }^{15}$ En nuestro estudio no observamos asociación del grado de displasia respecto al género.

En nuestra investigación, cinco de nueve muestras con DEO se localizaron en el borde lateral de lengua, lo cual puede relacionarse con zonas anatómicas que presentan mayor riesgo de desarrollar un carcinoma oral. ${ }^{16}$ Este hecho refuerza la necesidad de una explo- ración clínica minuciosa en tejidos blandos, colocando mayor énfasis en zonas tales como los bordes laterales de lengua cuando los pacientes cuentan con antecedentes de algún factor de riesgo para el desarrollo de cáncer. ${ }^{17}$ En la patogénesis del cáncer, la compleja interacción de los factores ambientales e intrínsecos, tales como los oncogenes $y /$ o genes supresores tumorales, es un amplio tema de estudio y análisis. ${ }^{5}$

P53 es una molécula que desempeña un papel importante en la carcinogénesis oral. Se han reportado mutaciones o algún mecanismo de inactivación que previene que el sistema de p53 actúe de manera correcta y en su lugar contribuye al proceso de malignización. Para detectar esas variantes, se pueden emplear métodos 


\section{Tabla 2: Distribución de la intensidad de inmunoexpresión en relación con el grado de displasia.*}

\begin{tabular}{lccc} 
& \multicolumn{3}{c}{ Grado de displasia } \\
\cline { 2 - 4 } & Leve & Moderada & Severa \\
\hline p53 convencional (DO-7) & & & \\
$\quad$ Negativo & 1 & 0 & 0 \\
Leve & 2 & 2 & 1 \\
Moderado & 0 & 0 & 1 \\
Intenso & 0 & 1 & 1 \\
p53 mutado (PAb 240) & & & \\
Negativo & 3 & 1 & 3 \\
Leve & 0 & 2 & 0 \\
$\quad$ Moderado & 0 & 0 & 0 \\
Intenso & 0 & 0 & 0 \\
\hline
\end{tabular}

* Expresión significativa $(\mathrm{p}<0.05)$. o con el proteasoma, respectivamente. ${ }^{9} \mathrm{Si}$ consideramos que dos de las nueve muestras fueron positivas a la forma mutada, significaría que sólo $22 \%$ podría contar con el potencial de transformación a un carcinoma. Los reportes sobre esta posibilidad varían respecto a la probabilidad, así como en el número de años de seguimiento; observando diversos reportes se podría considerar que el rango de transformación va de 11 a $36 \% .{ }^{21-24}$

\section{CONCLUSIONES}

Considerando la información anterior, nuestros resultados caen dentro de esa posibilidad, así como el de una transformación no lineal, ya que los casos fueron de severidad moderada. Por lo cual podríamos sugerir que, aunque es posible la detección inmunohistoquímica de las formas mutadas de p53, siempre será necesario detectar mecanismos de transformación adicionales a través de otros métodos o ensayos que nos revelen un mayor panorama de este proceso.

\section{AGRADECIMIENTOS}

moleculares, como la secuenciación génica, ensayos de PCR o bien la inmunohistoquímica. 6,18 La clona DO-7 es un anticuerpo capaz de detectar tanto a la proteína convencional o wild-type como la mutada, mientras la clona PAb 240 reconoce a la forma mutada de $\mathrm{p} 53 .{ }^{19}$ En nuestra publicación, ocho de nueve especímenes fueron positivos para p53 mediante la clona DO-7, lo cual sugeriría que su papel es importante en la patogénesis de la displasia; sin embargo, cuando analizamos su expresión mediante la clona PAb 240, sólo dos especímenes fueron positivos.

Diversos autores han reportado que la vida media y labilidad conformacional de la proteína p53 es muy corta, por lo que su detección en tejidos normales es compleja, aunque su inmunorreactividad puede ser observada en epitelios hiperplásicos y displásicos. ${ }^{19,20} \mathrm{~A}$ pesar de que nuestros resultados siguieren que la mayoría de las muestras fueron positivas a la clona DO-7, esto podría estar asociado con la propiedad de hiperproliferación de las displasias, lo cual refuerza el hecho de que estas lesiones cuentan con el potencial de convertirse en una neoplasia verdadera, debido a que poseen el potencial proliferativo que caracteriza al cáncer. No obstante, el observar sólo dos muestras positivas a la clona PAb 240, que detecta la forma mutada de p53, nos sugiere que la participación de esta molécula en el proceso de carcinogénesis epitelial temprana es más compleja, ya que sus cambios conformacionales pueden determinar su funcionamiento o degradación con la molécula MDM2
Investigación realizada gracias al programa UNAM-PAPIIT IN226720.

\section{REFERENCIAS}

1. Suwasini S, Chatterjee K, Purkait SK, Samaddar D, Chatterjee A, Kumar M. Expression of P53 protein and Ki-67 antigen in oral leukoplakia with different histopathological grades of epithelial dysplasia. J Int Soc Prev Community Dent. 2018; 8 (6): 513-522.

2. Wetzel SL, Wollenberg J. Oral potentially malignant disorders. Dent Clin North Am. 2020; 64 (1): 25-37.

3. Tilakaratne WM, Jayasooriya PR, Jayasuriya NS, De Silva RK. Oral epithelial dysplasia: Causes, quantification, prognosis, and management challenges. Periodontol 2000. 2019; 80 (1): 126-147.

4. Pallavi N, Nalabolu GRK, Hiremath SKS. Bcl-2 and c-Myc expression in oral dysplasia and oral squamous cell carcinoma: an immunohistochemical study to assess tumor progression. J Oral Maxillofac Pathol. 2018; 22 (3): 325-331.

5. Hanahan D, Weinberg RA. Hallmarks of cancer: the next generation. Cell. 2011; 144 (5): 646-674.

6. Vousden KH, Lane DP. p53 in health and disease. Nat Rev Mol Cell Biol. 2007; 8 (4): 275-283.

7. Manjunath S, Himadal CG, Divakar DD, Haleem S, Mohammad Faqeeh HA, Alshadidi MY. An immunohistochemical study of p53 expressions in oral submucous fibrosis. J Oral Maxillofac Pathol. 2019; 23 (2): 308.

8. Sirur DG, Tamgadge A, Tamgadge S, Bhalerao S, Gujjar PK. Correlation of p53 expression with histopathological and immunohistochemical features of human papillomavirus in oral leukoplakia. J Microsc Ultrastruct. 2020; 8 (3): 81-88.

9. Sasaki M, Nie L, Maki CG. MDM2 binding induces a conformational change in p53 that is opposed by heat-shock protein 90 and precedes p53 proteasomal degradation. J Biol Chem. 2007; 282 (19): 14626-14634. 
10. Aviso de privacidad integral. Facultad de Odontología, Universidad Nacional Autónoma de México. [Consultado en agosto de 2020] Disponible en: http://www.odonto.unam.mx/sites/default/files/ inline-files/1.AP Integral-Fac Esc Inst Centros\%20\%20 0.pdf

11. Trejo-Remigio DA- Jacinto-Alemán LF, Leyva-Huerta ER, NavarroBustos BR, Portilla-Robertson J. Ectodermal and ectomesenchymal marker expression in primary cell lines of complex and compound odontomas: a pilot study. Minerva Stomatol. 2019; 68 (3): 132-141.

12. Goodson ML, Sloan P, Robinson CM, Cocks K, Thomson PJ. Oral precursor lesions and malignant transformation--who, where, what, and when? Br J Oral Maxillofac Surg. 2015; 53 (9): 831-845.

13. López Jornet P, Saura Ingles A, Cozar Fernández A. Estudio de las lesiones precancerosas de la mucosa bucal en el paciente geriátrico. Rev Esp Geriatr Gerontol. 1999; 34 (3): 163-171.

14. Singh S, Singh J, Chandra S, Samadi FM. Prevalence of oral cancer and oral epithelial dysplasia among North Indian population: A retrospective institutional study. J Oral Maxillofac Pathol. 2020; 24 (1): $87-92$

15. Gaitán-Cepeda LA, Peniche-Becerra AG, Quezada-Rivera D. Trends in frequency and prevalence of oral cancer and oral squamous cell carcinoma in Mexicans. A 20 years retrospective study. Med Oral Patol Oral Cir Bucal. 2011; 16 (1): e1-5.

16. Aittiwarapoj A, Juengsomjit R, Kitkumthorn N, Lapthanasupkul P. Oral potentially malignant disorders and squamous cell carcinoma at the tongue: clinicopathological analysis in a Thai population. Eur J Dent. 2019; 13 (3): 376-382.

17. Nagata G, Santana T, Queiroz A, Caramez RH, Trierveiler M. Evaluation of epithelial dysplasia adjacent to lip squamous cell carcinoma indicates that the degree of dysplasia is not associated with the occurrence of invasive carcinoma in this site. J Cutan Pathol. 2018; 45: 647-651.

18. Gonzalez-Moles MA, Gil-Montoya JA, Ruiz-Avila I, Esteban F, Bascones-Martinez A. Differences in the expression of p53 protein in oral lichen planus based on the use of monoclonal antibodies DO7 and PAb 240. Oral Oncol. 2008; 44 (5): 496-503.

19. Silva Júnior JA, Camisasca DR, Bernardo V, Ribeiro GS, Dias FL, de Faria PA et al. The significance of p53 immunoexpression with different clones (DO-7 and PAb-240) in oral squamous cell carcinoma. ORL J Otorhinolaryngol Relat Spec. 2013; 75 (2): 82-90.
20. Piffkó J, Bánkfalvi A, Ofner D, Berens A, Tkotz T, Joos U et al. Expression of p53 protein in oral squamous cell carcinomas and adjacent non-tumorous mucosa of the floor of the mouth: an archival immunohistochemical study using wet autoclave pretreatment for antigen retrieval. J Oral Pathol Med. 1995; 24 (8): 337-342.

21. Mincer HH, Coleman SA, Hopkins KP. Observations on the clinical characteristics of oral lesions showing histologic epithelial dysplasia. Oral Surg. 1972; 33 (3): 389-392.

22. Pindborg JJ, Daftary DK, Mehta FS. A follow-up study of sixty-one oral dysplastic precancerous lesions in Indian villagers. Oral Surg. 1977; 43 (3): 383-390.

23. Silverman S Jr, Gorsky M, Lozada F. Oral leukoplakia and malignant transformation. A follow-up study of 257 patients. Cancer. 1984; 53 (3): 563-568.

24. Warnakulasuriya S, Kovacevic T, Madden P, Coupland VH, Sperandio $\mathrm{M}$, Odell $\mathrm{E}$ et al. Factors predicting malignant transformation in oral potentially malignant disorders among patients accrued over a 10year period in South East England. J Oral Pathol Med. 2011; 40 (9): 677-683.

Conflicto de intereses: Los autores declaran no presentar conflicto de intereses.

Aspectos éticos: Esta investigación se condujo bajo todos los lineamientos éticos correspondientes al Reglamento de la Ley General de Salud en Materia de Investigación para la Salud, respetando los derechos de privacidad acorde al Aviso de privacidad integral, Facultad de Odontología, Universidad Nacional Autónoma de México.

Financiamiento: Investigación realizada gracias al programa UNAM-PAPIIT IN226720.

Correspondencia:

Luis Fernando Jacinto-Alemán

E-mail: jacintoalemánlf@gmail.com 